\title{
Sweat Biomarker Sensor Incorporating Picowatt, Three-Dimensional Extended Metal Gate Ion Sensitive Field Effect Transistors
}

Junrui Zhang ${ }^{1 *}$, Maneesha Rupakula ${ }^{1}$, Francesco Bellando ${ }^{1}$, Erick Garcia Cordero ${ }^{1}$, Johan Longo ${ }^{2}$, Fabien Wildhaber ${ }^{2}$, Guillaume Herment ${ }^{1}$, Hoël Guérin ${ }^{2}$, Adrian Mihai Ionescu ${ }^{1}$

${ }^{1}$ Nanoelectronic Devices Laboratory, Ecole Polytechnique Fédérale de Lausanne, Lausanne 1015, Switzerland

${ }^{2}$ Xsensio SA, Innovation Park, Lausanne 1015, Switzerland

*corresponding author: junrui.zhang@epfl.ch 


\section{Supplementary information}

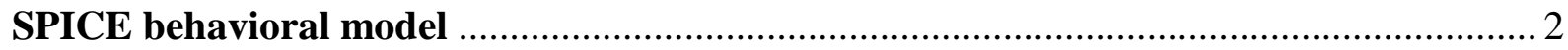

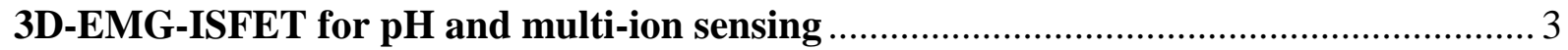

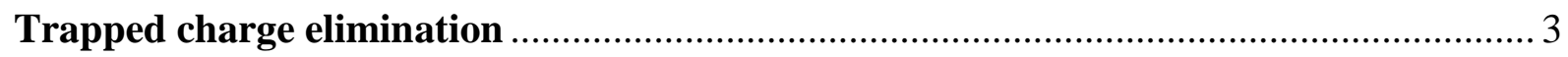

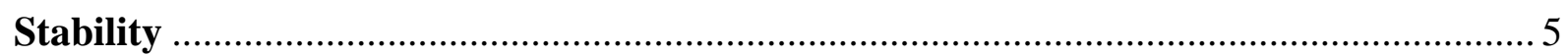

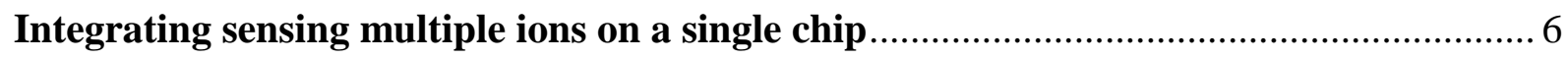

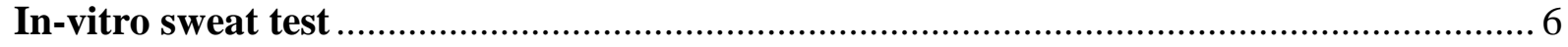

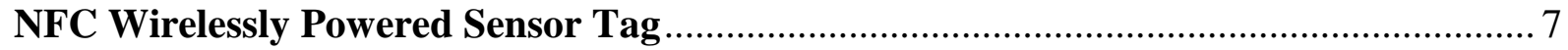

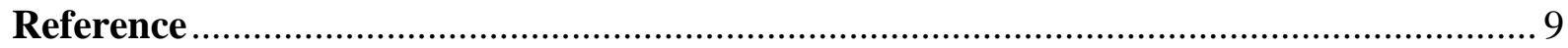

\section{SPICE behavioral model}

A SPICE behavioral model $[1,2]$, sketched in Fig. S1, is used to simulate the $\mathrm{pH}$ sensing characteristics of the 3D-EMG-ISFET. The simulation results cohere with the measurement results as shown in Fig. S2a. In the SPICE model, the ISFET is recognized as a series combination of two parts: solution + semiconductor. The liquid gate $\left(G^{\prime}\right)$ potential is affected by both $\mathrm{pH}$ and $\mathrm{V}_{\text {ref, }}$ through Equations $(\mathrm{S} 1)-(\mathrm{S} 2)$.

$$
\begin{aligned}
& V_{G}^{\prime}=V_{\text {ref }}-V_{\text {Chem }} \\
& V_{\text {Chem }}=\gamma+2.303 \alpha \frac{k T}{q} p H
\end{aligned}
$$

In which $\gamma$ is a grouping of chemically and physically related constant potentials that includes the interface potentials at the reference eletrode. $\alpha$ is a dimensionless partitioning parameter $(0<\alpha<1)$ reflecting the influence of buffer capacity for protons of the sensing dielectric surface and the equivalent capacitance of the electrical double layer $\left(\mathrm{C}_{\text {Gouy }}, \mathrm{C}_{\text {Helm }}\right)$. $\mathrm{k}$ is the Boltzmann constant. $\mathrm{T}$ is the absolute temperature. $\mathrm{q}$ is the elementary charge.

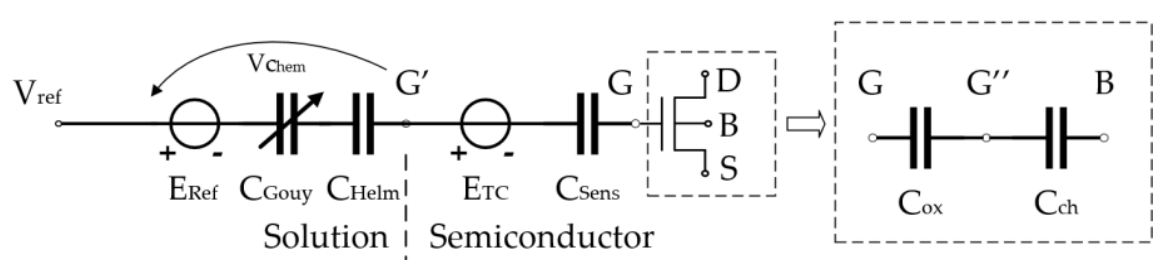

Fig. S1 Schematic of ISFET model applied to simulate the $\mathrm{pH}$ sensing characteristics. $\mathrm{E}_{\mathrm{TC}}$ : voltage drop due to trapped charge; $\mathrm{E}_{\text {ref: }}$ potential difference at reference electrode to solution interface. Inset: simplified capacitance divider to model the MOSFET.

In CMOS process, charges are inevitably trapped in the gate insulator as well as the passivation layers. Modeling the influence of trapped charges as a constant DC offset (ETC), the resulting ISFET model exhibits a gate voltage $\left(\mathrm{V}_{\mathrm{G}}\right)$ that is characterized by Equation $(\mathrm{S} 3)$ [1].

$$
V_{G}-V_{B}=\left(V_{\text {ref }}-\gamma-2.303 \alpha \frac{k T}{q} p H-E_{T C}\right) \frac{\mathrm{C}_{\text {Sens }}}{\mathrm{C}_{\text {Sens }}+\left(\mathrm{C}_{o x} \mathrm{C}_{c h} /\left(\mathrm{C}_{o x}+\mathrm{C}_{c h}\right)\right)}
$$

Where, $\mathrm{C}_{\text {Sens }}$ is the capacitance of the sensing dielectric. $\mathrm{C}_{o x}$ is the underlying MOSFET's gate insulator capacitance. $\mathrm{C}_{c h}$ is the channel depletion capacitance. The voltage divider effects of 
$\mathrm{C}_{\text {Gouy }}$ and $\mathrm{C}_{\text {Helm }}$ are negelected due to the fact that $\mathrm{C}_{\text {Gouy }}, \mathrm{C}_{\text {Helm }}$ are orders of magnitude larger than $\mathrm{C}_{\text {sens }}, \mathrm{C}_{o x}$, and $\mathrm{C}_{d}$.

\section{D-EMG-ISFET for $\mathrm{pH}$ and multi-ion sensing}

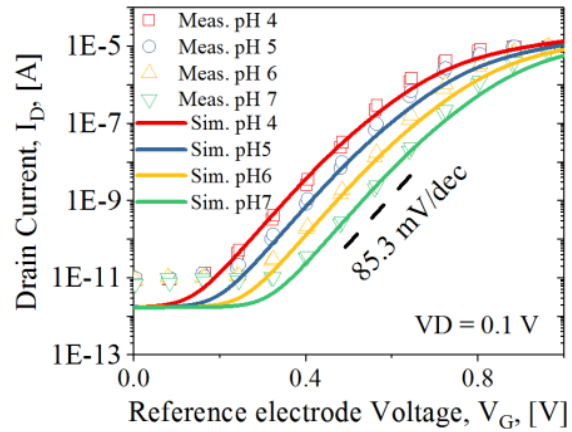

(a)

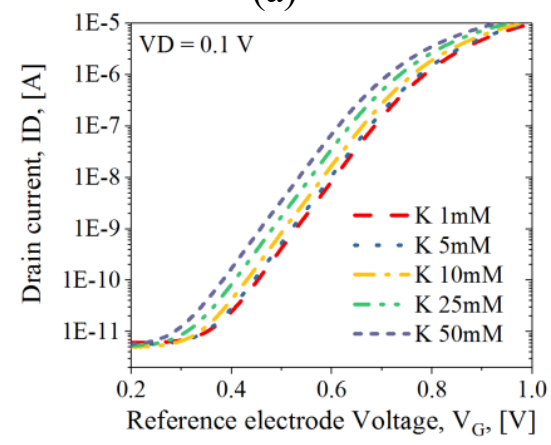

(c)

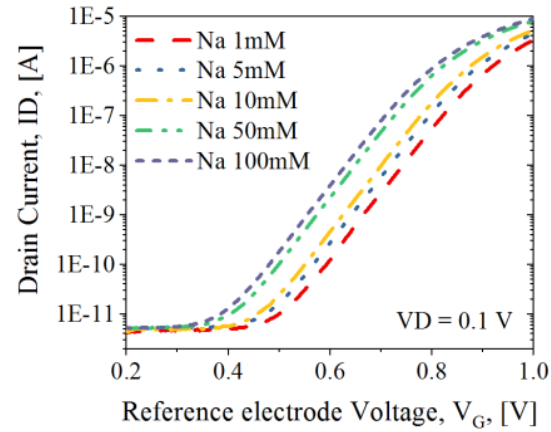

(b)

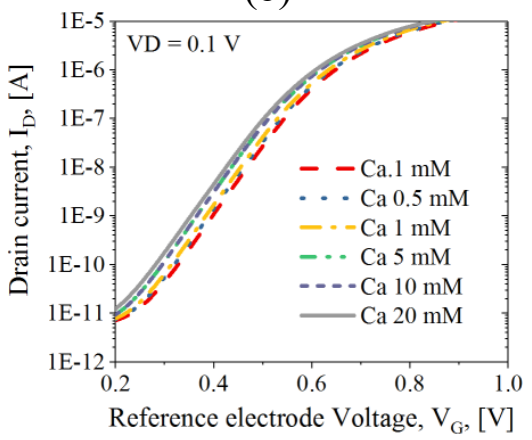

(d)

Fig. S2 a) $\mathrm{I}_{\mathrm{D}} \mathrm{V}_{\mathrm{G}}$ Characteristics of the $3 \mathrm{D}$-EMG-ISFET in various $\mathrm{pH}$ buffers. SPICE behavioral model [2] simulations agree well with measurements. b) $\mathrm{I}_{\mathrm{D}} \mathrm{V}_{\mathrm{G}}$ characteristics of the $3 \mathrm{D}-\mathrm{EMG}-\mathrm{Na}^{+}$sensitive $\mathrm{FET}$, in $\mathrm{NaCl}$ solutions of various concentrations. c) $\mathrm{I}_{\mathrm{D}} \mathrm{V}_{\mathrm{G}}$ characteristics of the $3 \mathrm{D}-\mathrm{EMG}-\mathrm{K}^{+}$sensitive $\mathrm{FET}$, in $\mathrm{KCl}$ solutions of various concentrations. d) $\mathrm{I}_{\mathrm{D}} \mathrm{V}_{\mathrm{G}}$ characteristics of the $3 \mathrm{D}-\mathrm{EMG} \mathrm{Ca}^{2+}$ sensitive $\mathrm{FET}$, in $\mathrm{CaCl}_{2}$ solutions of various concentrations.

\section{Trapped charge elimination}

The accurate predictability of sensor performance is a key to its wide application. As introduced before, charges are inevitably trapped in the gate insulator and the passivation layers during the CMOS process. Seen from Equation S3, $\mathrm{E}_{T C}$ and $\mathrm{C}_{\text {Sens }}$ are the two process dependent parameters, which determine the accuracy of the ISFET macromodel. They are studied in the following paragraphs.

In order to know exactly the sensing dielectric composition, two sets of Transmission Electron Microscopy (TEM) images are taken, Figs. S3b - S3g and Figs. S4b - S4d, showing the composition of material layers over the top metal gate, for devices before (Fig. S3a) and after (Fig. S4a) the RIE steps, respectively. A comparison between these two sets of TEM images clearly shows that there is a layer of $7 \mathrm{~nm} \mathrm{Al}_{2} \mathrm{O}_{3}$ formed after exposing the $\mathrm{Al}$ top metal with the RIE steps.

An observation of Figs. S3a and S4a show exactly the same FEOL devices, with the same stacks of metal and via layers, interspersed with IMDs, whereas the only difference is in the last passivation layers at the BEOL. 


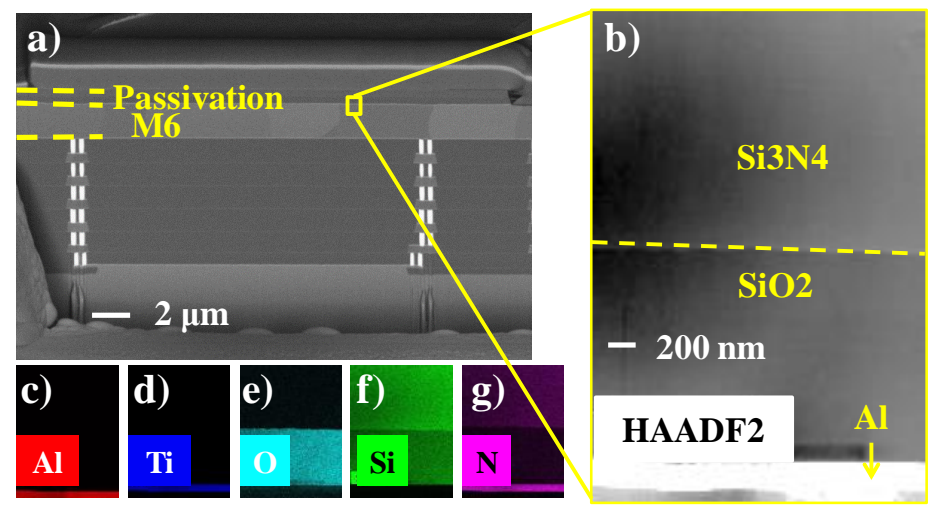

Fig. S3 a) FIB Cross section of CMOS ISFET before RIE, b) HAADF STEM indicating passivation Si3N4/SiO2 layers above the top metal. c) - g) EDX analysis confirm constituent elements of $\mathrm{Al}, \mathrm{Ti}, \mathrm{O}, \mathrm{Si}, \mathrm{N}$ in the cross section.
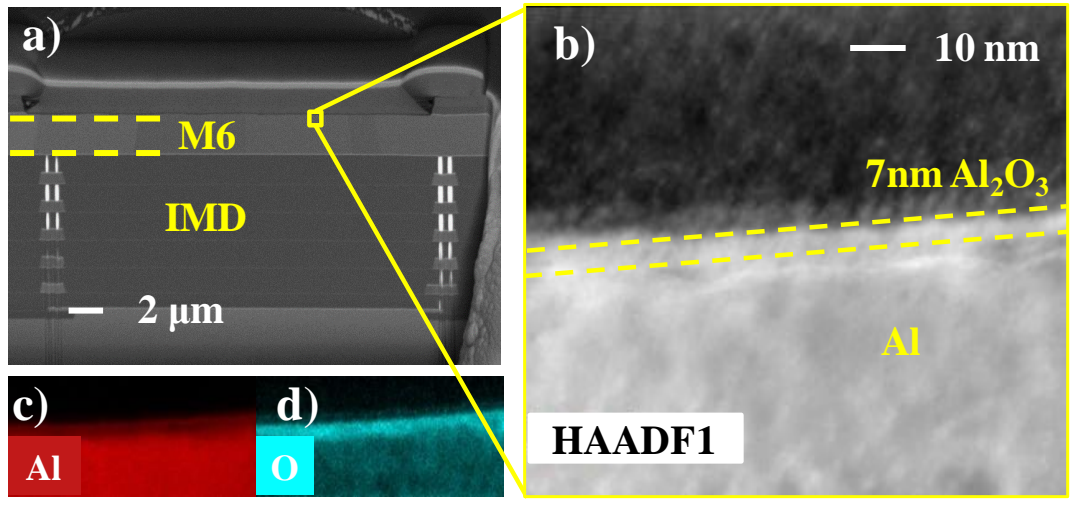

Fig. S4 a) FIB cross section of 3D-EMG-ISFET with RIE etched openings. b) HAADF STEM image indicating $7 \mathrm{~nm}$ native oxide on exposed top metal used for sensing. c) - d) EDX analysis confirms elemental composition of $\mathrm{Al}_{2} \mathrm{O}_{3}$.

In Fig. S3, it is obvious that the conventional CMOS ISFET, which utilizes the $\mathrm{Si}_{3} \mathrm{~N}_{4}$ layer as the sensing dielectric, suffers from trapped charge in the $\mathrm{Si}_{3} \mathrm{~N}_{4}$ and $\mathrm{SiO}_{2}$ passivation layers. This has been reported by $[1,3]$.

In the adopted $0.18 \mu \mathrm{m}$ CMOS foundry process, the Front-End-of-Line (FEOL) is well controlled, resulting in MOSFET devices with a measured threshold voltage variation $\left(\Delta \mathrm{V}_{\text {th }}\right)$ range of $12 \mathrm{mV}$. This gave us a hint of trying to remove the passivation layers completely (Fig. $\mathrm{S} 4)$, in order to eliminate the trapped charge.

Shown in Fig. S5, are results from a statistical study of 4 devices in different chips, the CMOS ISFETs which utilize the Si3N4 passivation layer for sensing (Fig. S3a), exhibit a $\Delta \mathrm{V}_{\text {th }}$ of 4.39 $\mathrm{V}$. The 3D-EMG-ISFETs reduced this spread to $184 \mathrm{mV}$, due to removal of the passivation layers, together with charges trapped in them during the foundry process. The wet etching step, described in the methods section, further squeezed this span to $56 \mathrm{mV}$. 


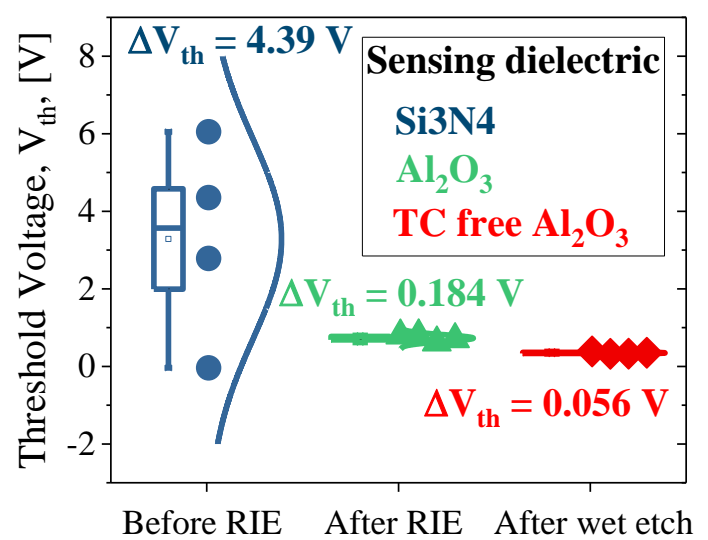

Fig. S5 $\mathrm{V}_{\text {th }}$ spread in $\mathrm{pH} 7$ buffer solution. Before RIE: the $\mathrm{Si}_{3} \mathrm{~N}_{4}$ passivation layer is used as sensing dielectric. After RIE: the $\mathrm{Al}_{2} \mathrm{O}_{3}$ layer is used for sensing. After wet etch: trapped charge free (TC free) $\mathrm{Al}_{2} \mathrm{O}_{3}$ is used for sensing.

\section{Stability}

Long term stability is a key figure of merit for sensors. ISFET sensors' threshold voltage undergoes drift due to surface potential changes caused by interactions between the liquid and sensing dielectric. And the stability of an ISFET sensor is characterized by the drift rate of its threshold voltage (unit: $\mathrm{mV} / \mathrm{h}$ ). It can only be measured indirectly [4]: one of the ways is to bias the ISFET's terminals at constant voltages, and record the time dependent behavior of $I_{D}$. The $V_{\text {th }}$ drift rate can be converted from $I_{D}$ drift by using the $I_{D} V_{G}$ characteristic curve.

Two kinds of 3D-EMG-ISFET sensors are compared in terms of long term stability. They are different only in the sensing dielectric: 1 . native oxide $\left(7 \mathrm{~nm} \mathrm{Al}_{2} \mathrm{O}_{3}\right)$, 2. controlled oxide (7 $\mathrm{nm}$ native $\mathrm{Al}_{2} \mathrm{O}_{3}+8 \mathrm{~nm}$ Atomic Layer Deposited $\mathrm{HfO}_{2}$ ). The 3D-EMG-ISFETs are immersed in $\mathrm{pH} 4$ buffer solution with constant bias voltages, while $\mathrm{I}_{\mathrm{D}}$ is monitored from the first minute that the sensing area gets in contact with the liquid. Shown in Fig. S6a is the $I_{D}$ drift of the 3DEMG-ISFET in a 20 hours long term measurement. As have been exhaustively reported by other researchers $[5,6]$, the rapid decreasing of $I_{D}$ in the first hours, is due to the hydroxyl group diffusion in the oxide. After the $14^{\text {th }}$ hour, a drift rate of $0.67 \mathrm{mV} / \mathrm{h}$ is calculated for the ISFET with native oxide, whereas $2 \mathrm{mV} / \mathrm{h}$ is extracted for the ISFET with controlled oxide for the same period of time (Fig. S6b). This comparison shows that the native oxide layer manifests itself as a qualified sensing layer.

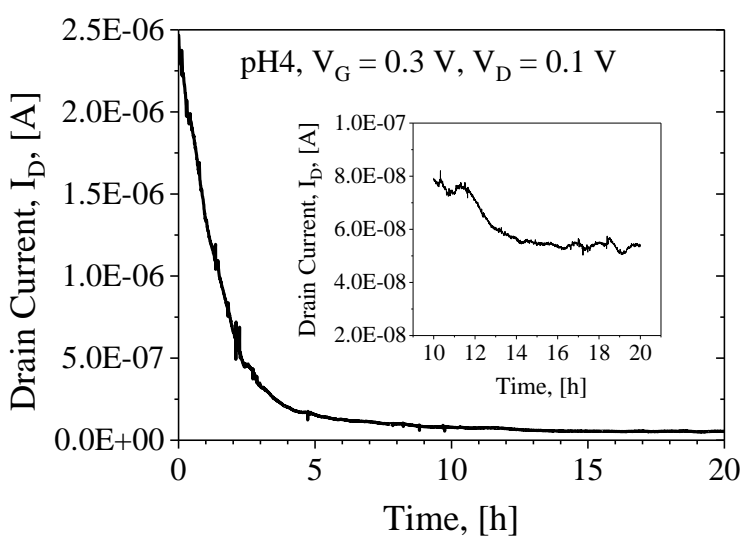

(a)

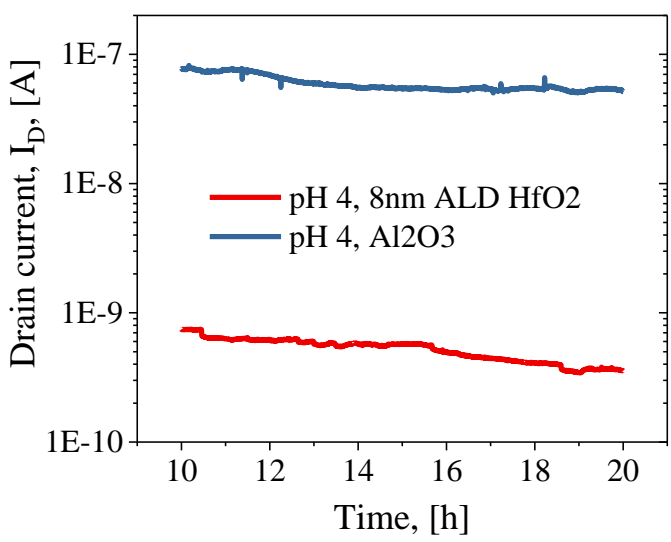

(b)

Fig. S6 a) Long term $\mathrm{I}_{\mathrm{D}}$ drift of the 3D-EMG-ISFET with $\mathrm{Al}_{2} \mathrm{O}_{3}$ as sensing layer. Inset: zoom in of the drift plot from $10 \mathrm{~h}$ to $20 \mathrm{~h}$. b) $\mathrm{I}_{\mathrm{D}}$ drifts comparison between 3D-EMG-ISFETs with $\mathrm{Al}_{2} \mathrm{O}_{3}$ and ALD layer of $\mathrm{HfO}_{2}$. Converted drift rate: $0.67 \mathrm{mV} / \mathrm{h}-\mathrm{Al}_{2} \mathrm{O}_{3}, 2 \mathrm{mV} / \mathrm{h}-\mathrm{HfO}_{2}$. 


\section{Integrating sensors for multiple ions on a single chip}

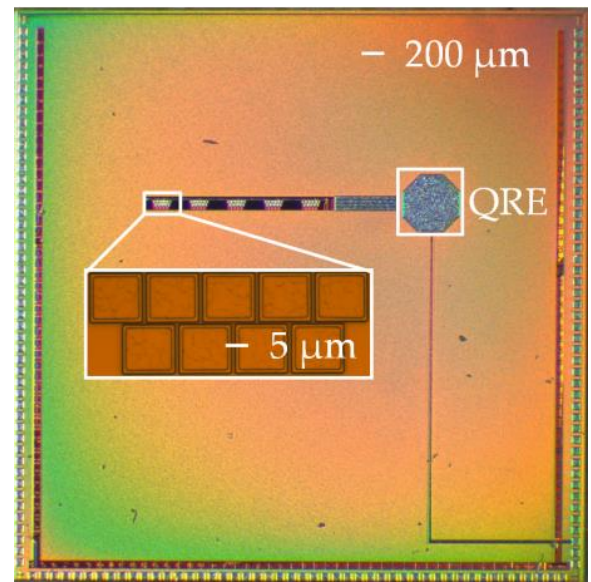

(a)

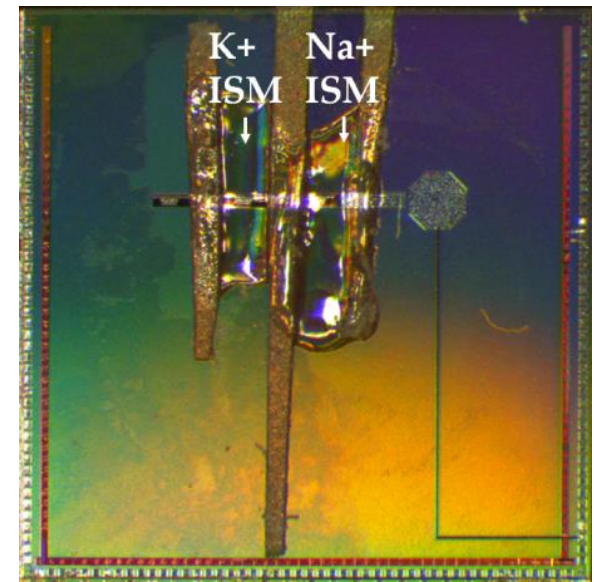

(b)

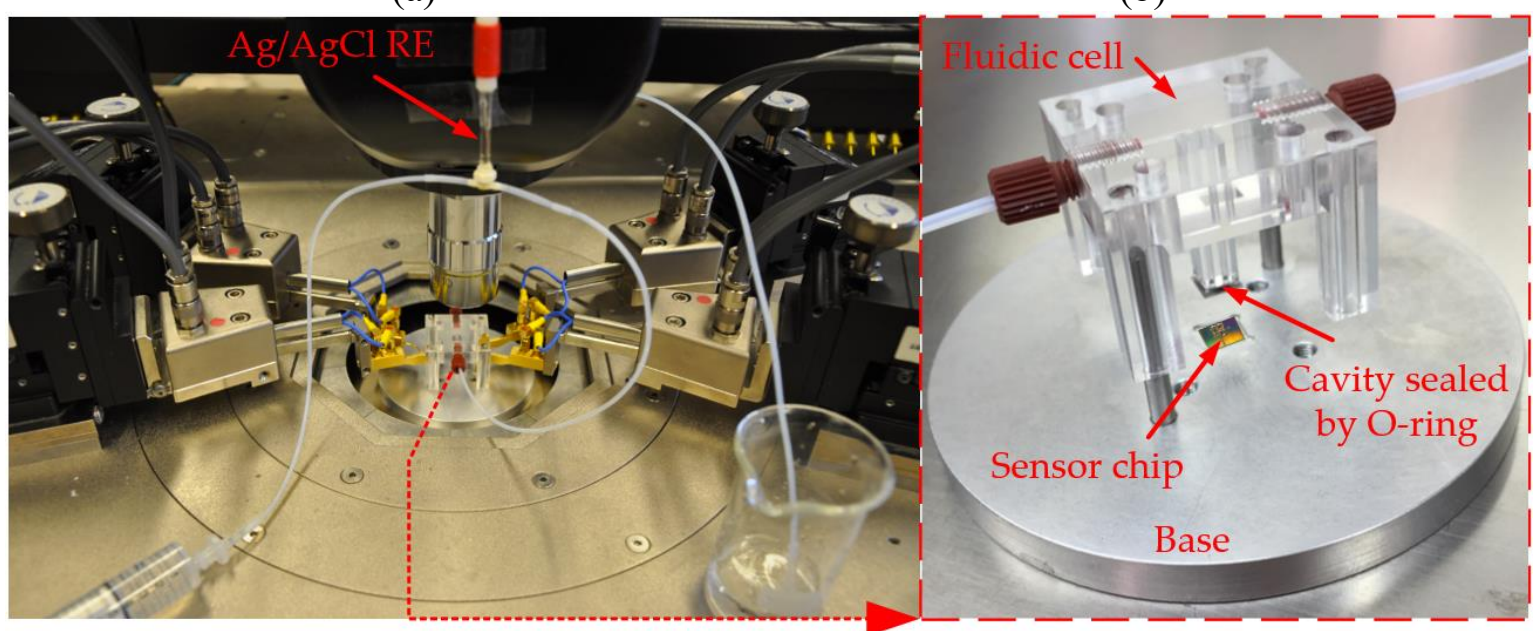

(c)

Fig. S7 a) CMOS sensor chip containing five groups of 3D-EMG-ISFET sensors. QRE (quasi reference electrode): will be introduced later. b) A drop of $\mathrm{K}^{+}$and $\mathrm{Na}^{+}$ISM is casted on top of two groups of ISFET, respectively. c) Measurement setup for simultaneously measuring two different ion sensors in the CMOS sensor chip shown in a), Inset: Exploded view of the fluidic cell.

\section{In-vitro sweat test}

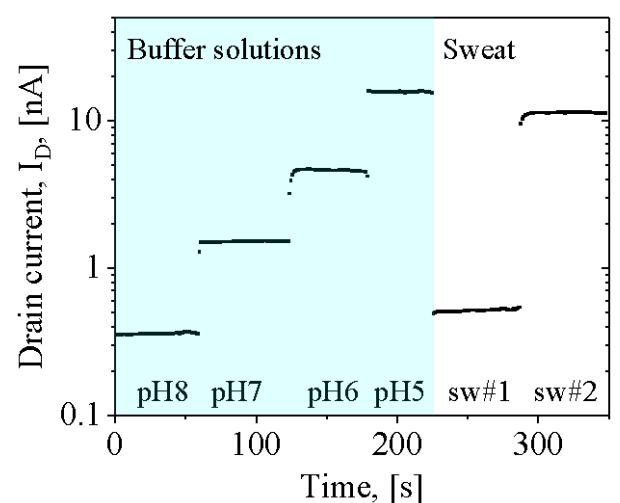

(a)

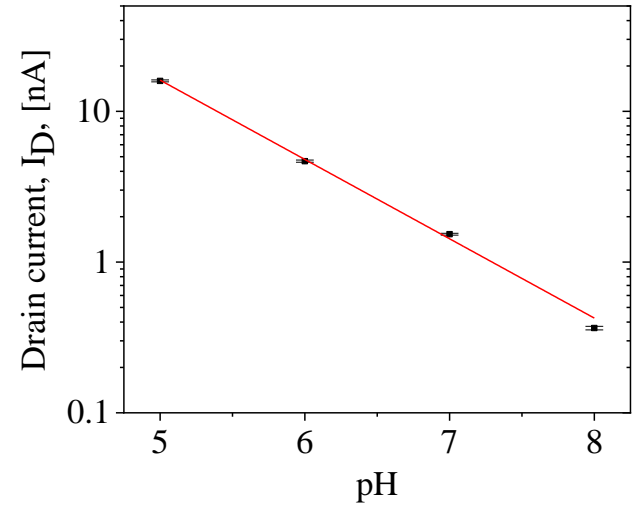

(b) 


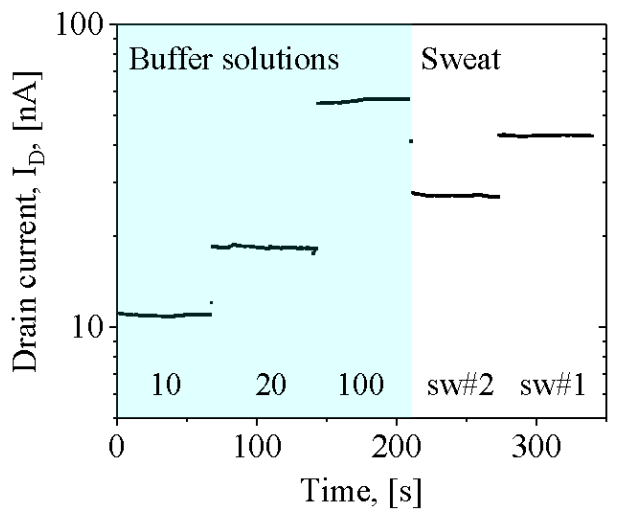

(c)

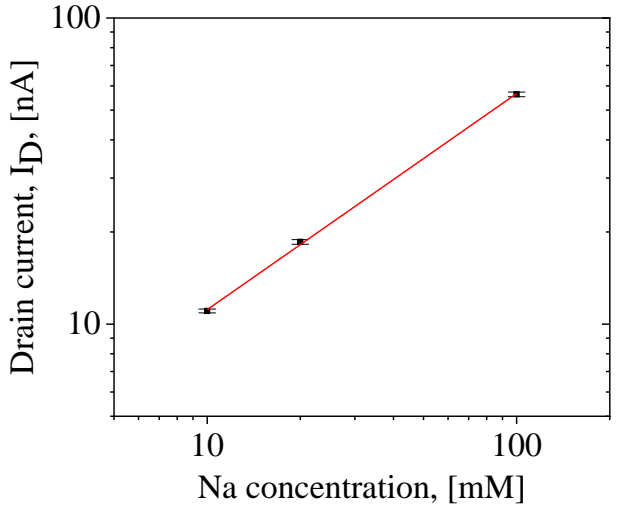

(d)

Fig. S8 a) Dynamic measurements with sweat samples and various $\mathrm{pH}$ buffers to extract $\mathrm{b}$ ) calibration curve for 3D-EMG-pH sensors, and calculate sweat $\mathrm{pH}$ values. c) Dynamic measurements with sweat samples and various $\mathrm{NaCl}$ solutions to extract d) calibration curve for 3D-EMG- $\mathrm{Na}^{+}$sensors, and calculate sweat $\mathrm{Na}^{+}$concentrations. Error bars showing the standard deviation from the same sensor during the period of dynamic measurements.

\section{NFC Wirelessly Powered Sensor Tag}

As shown in Fig. 1d, the ASIC developed for multiplexed readout of different sensors contains an ISFET array, a 16:1 multiplexer, and an active integrator. The multiplexer is consisted of 16 CMOS switches and a 4-to-16 digital decoder, shown in Fig. S9a. The digital inputs of the decoder are terminals A, B, C, D. Digital codes are sent to these terminals through the general purpose in/out (GPIO) of the microcontroller unit (MCU). The digital inputs are decoded into a 16 bit digital output code $(\mathrm{C} 0, \mathrm{C} 1, \ldots, \mathrm{C} 15)$, among which there is always one bit and only one bit is a digital ' 1 ', other bits are digital ' 0 '. Each of the digital outputs is connected to a single pole double throw (SPDT) CMOS switch, as shown in Fig. S9b. When Ci outputs a digital ' 0 ', the corresponding drain terminal of the ISFET $i$ is connected to ground through the switch. When Ci output a digital ' 1 ', the drain terminal of the ISFET $i$ is connected to the active integrator, which provides DC bias voltage equal to VD to the drain terminal, as well as outputs an analog voltage Vout.

$$
V_{\text {out }}-V_{D}=\frac{1}{C_{\text {int }}} \int_{t=0}^{T_{C L K}} I_{D} d t
$$

Therefore, with the values of $C_{\text {int }}, V_{D}, V_{\text {out }}$, and the period of the CLK signal applied to the integration switch, the current value of $I_{D}$ is derived.

Since the integrator type of readout circuitry rules out a large percentage of the white noise, it is very useful in improving the signal to noise ratio. It is especially helpful in reducing noise introduced by communication channels, since a large amount of random processes that occur in nature add to the white noise in the channels. [7] 


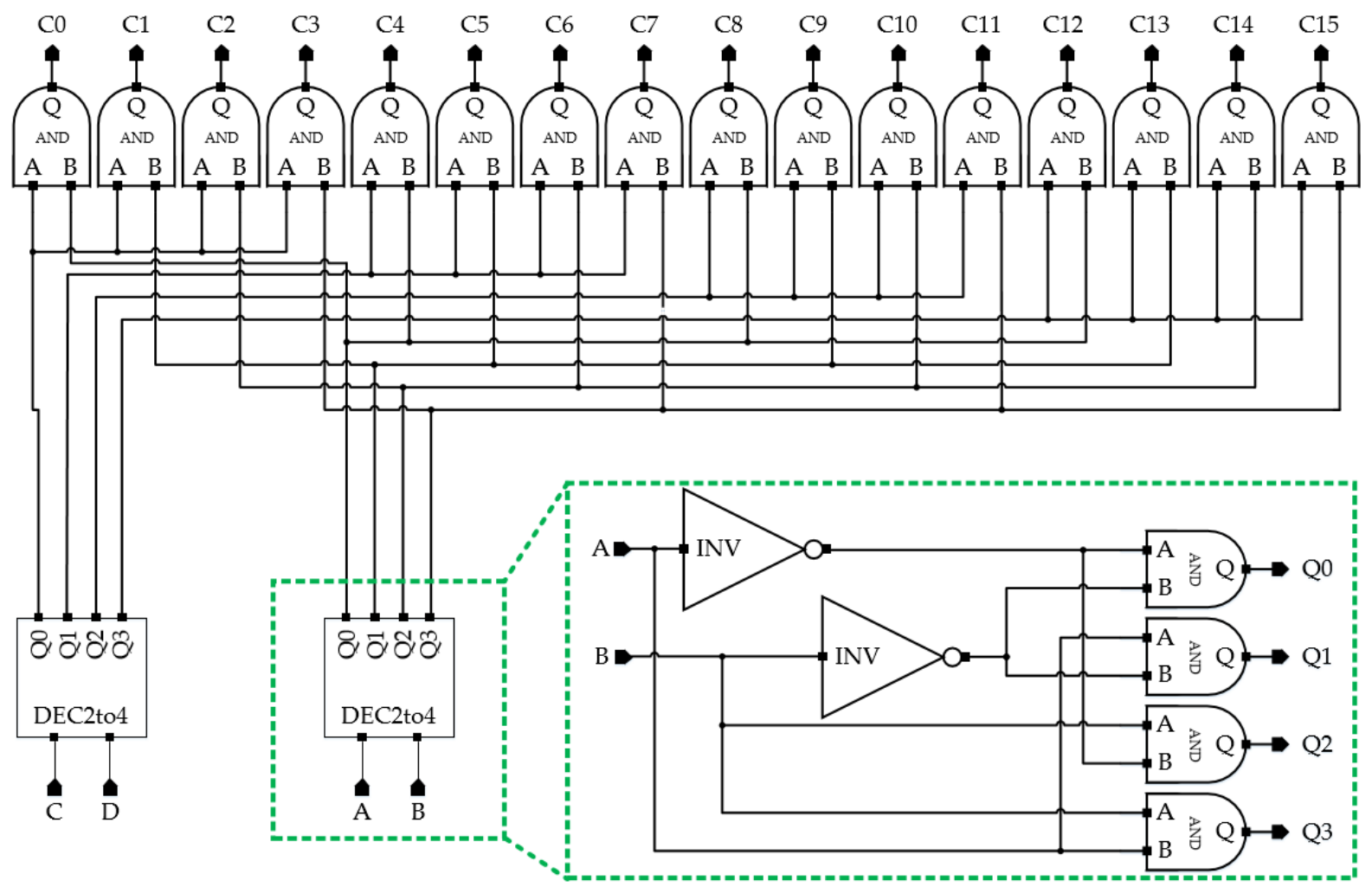

(a)

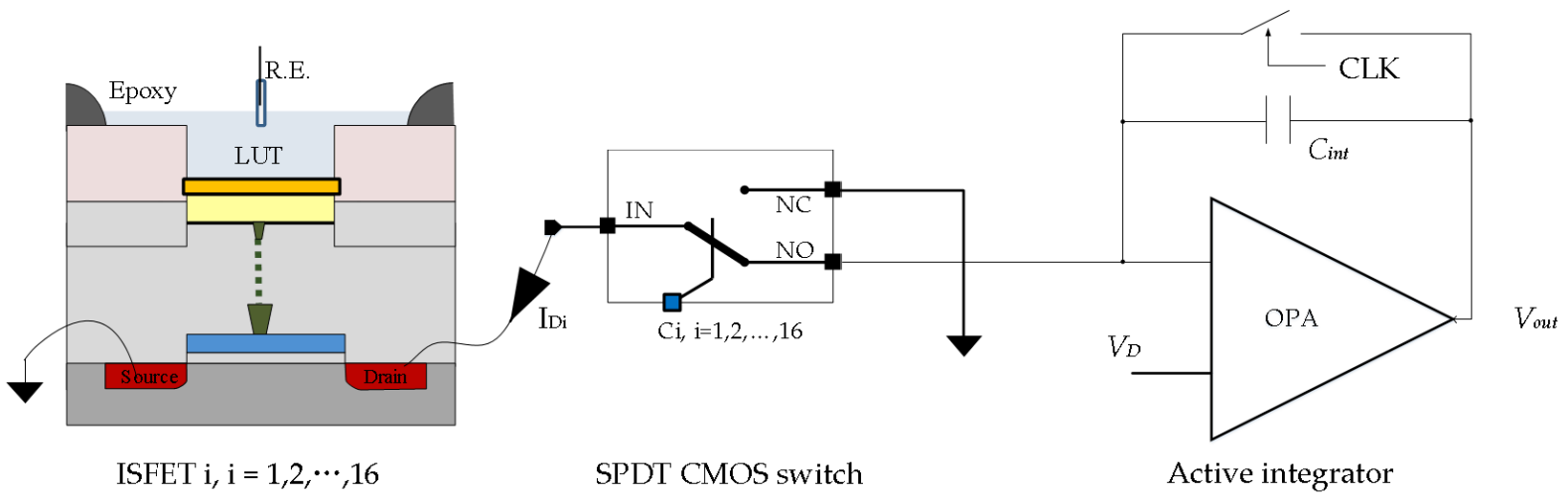

(b)

Fig. S9. a) 4-to-16 decoder, as a crucial component of the 16:1 multiplexer. With the multiplexer, the ASIC is able to switch among 16 different ISFET sensors with 4 GPIO digital control signals. b) Schematic of the readout circuitry for when one of the ISFETs is switched on and connected to the active integrator.

As introduced in Fig. 1, the NFC platform has an analog to digital converter, which is dedicated to converting the Vout into digital signal and not shown in Fig. S9. The digitized Vout is then transmitted through NFC communication to the mobile application. From the mobile application, $I_{D}$ is calculated with the aid of Equation S4. Now we are able to record the drain current with the NFC platform and a dedicated android application. Chronoamperometric response has been recorded by the NFC platform (Fig. 1a of the main text), in order to justify its wirelessly powered, multiplexed sensing capability. The 3D-EMG-ISFET for $\mathrm{pH}$ sensing, is sequentially immersed in drops of $\mathrm{pH}$ buffer. Time dependent measurement of the drain current $\left(\mathrm{I}_{\mathrm{D}}\right)$ is recorded both with a Precision Semiconductor Parameter Analyzer (HP 4156), and with the NFC platform. A good agreement is shown in Fig. S10a. The same has been done with the 
3D-EMG-Na ${ }^{+}$sensitive FET, and plotted in Fig. S10b, demonstrating the NFC platform's accurate sensing capability.

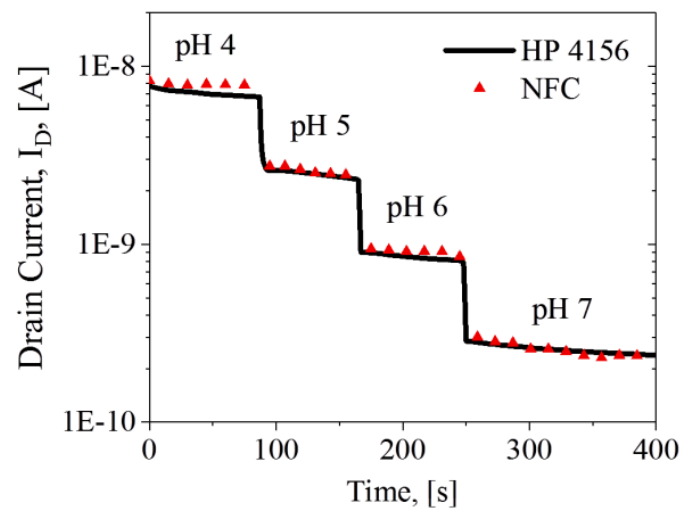

(a)

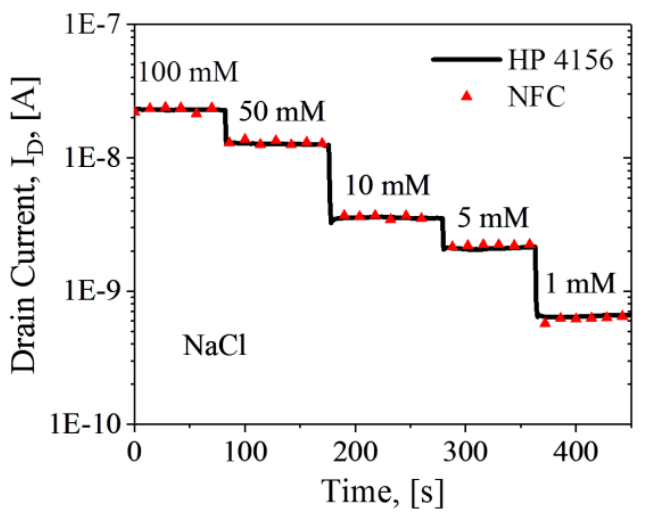

(b)

Fig. S10 Chronoamperometric readout of the $\mathrm{pH}$ sensing 3D-EMG-ISFET (a), and 3D-EMG-Na ${ }^{+}$sensitive FET (b), using NFC communication vs. with Precision semiconductor parameter analyzer (HP 4156).

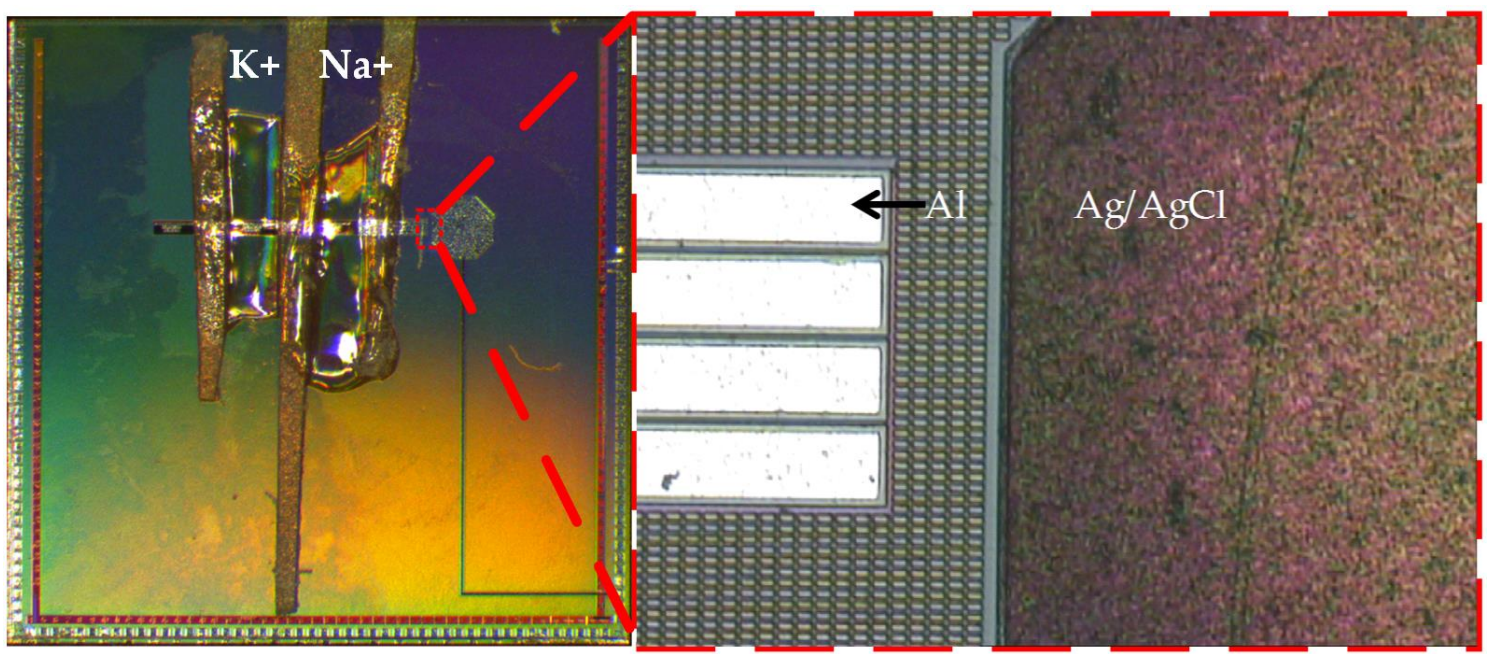

Fig. S11 Miniaturized $\mathrm{Ag} / \mathrm{AgCl}$ quasi reference electrode (QRE). The octagonal shape shown on the left was originally fabricated in the foundry, in the top metal layer which is Aluminum (Al). After the post-process introduced in the methods section, the electrode is turned into an $\mathrm{Ag} / \mathrm{AgCl} \mathrm{QRE}$.

\section{Reference}

[1] P. Georgiou, C. Toumazou, "ISFET characteristics in CMOS and their application to weak inversion operation," in Sensors and Actuators B 2009, 143(1): 211-217.

[2] S. Martinoia, G. Massobrio, "A behavioral macromodel of the ISFET in SPICE," in Sensors and Actuators B 2000, 62(3): 182-189.

[3] P.A.Hammond, D.R. S.Cumming, "Performance and system-on-chip integration of an unmodified CMOS ISFET," in Sensors and Actuators B: Chemical 2005, 111-112: 254-258.

[4] S. Jamasb, "An analytical technique for counteracting drift in ion-selective field effect transistors (ISFETs)," in IEEE Sensors Journal, vol. 4, no. 6, pp. 795-801, Dec. 2004. 
[5] TADAYUKI MATSUO and MASAYOSHI ESASHI, "Methods of ISFET fabrication," in Sensors and Actuators 1981, 1: 77-96.

[6] S. Jamasb, S. Collins, R. L. Smith, "A physical model for drift in pH ISFETs," in Sensors and Actuators B: Chemical 1998, 49(1-2): 146-155.

[7] McClaning, K., Vito, T. Radio Receiver Design. (Noble Publishing Corporation, 2000). 